\title{
KORGAN: An Efficient PKI Architecture Based on PBFT Through Dynamic Threshold Signatures
}

\author{
Murat Yasin Kubilay ${ }^{1,3}$, Mehmet Sabir Kiraz ${ }^{2,4}$, Haci Ali Mantar ${ }^{1}$ \\ 1 Department of Computer Engineering, Gebze Technical University, Kocaeli, Turkey, \\ 2 De Montfort University, School of Computer Science and Informatics, Leicester, UK \\ ${ }^{3}$ Deutsche Bank, Eschborn, Germany \\ 4 NChain, London, UK \\ muratkubilay@gtu.edu.tr, m.kiraz@gmail.com, hamantar@gtu.edu.tr
}

\begin{abstract}
During the last decade, several misbehaving Certificate Authorities (CA) have issued fraudulent TLS certificates allowing MITM kinds of attacks which result in serious security incidents. In order to avoid such incidents, Yakubov et al. recently proposed a new PKI architecture where CAs issue, revoke, and validate X.509 certificates on a public blockchain. However, in their proposal TLS clients are subject to MITM kinds of attacks and certificate transparency is not fully provided.

In this paper, we eliminate the issues of the Yakubov et al.'s scheme and propose a new PKI architecture based on permissioned blockchain with PBFT consensus mechanism where the consensus nodes utilize a dynamic threshold signature scheme to generate signed blocks. In this way, the trust to the intermediary entities can be completely eliminated during certificate validation. Our scheme enjoys the dynamic property of the threshold signature because TLS clients do not have to change the verification key even if the validator set is dynamic. We implement our proposal on private Ethereum network to demonstrate the experimental results. The results show that our proposal has negligible overhead during TLS handshake. The certificate validation duration is less than the duration in the conventional PKI and Yakubov et al.'s scheme.
\end{abstract}

Keywords: SSL/TLS, PKI, Certificate Transparency, PBFT, Dynamic Threshold Signatures

\section{Introduction}

TLS is the most widely used cryptographic protocol in today's internet for secure communications [7]. The main purpose of TLS is to provide end-toend security between client/server applications to prevent Man-In-The-Middle (MITM) kinds of attacks such as eavesdropping, tampering, or message forgery so that a malicious third party secretly cannot intercept, change or modify the communication traffic [6]. TLS enables establishment of a secure channel that ensures authentication, confidentiality and integrity between the communicating 
peers. Authentication and key establishment take place in the handshake protocol phase of TLS. X.509 certificates are used to verify the authenticity of the peers which are issued, revoked, and managed under a set of policies, roles, and cryptographic methods which have been modelled under the so-called Public Key Infrastructure (PKI). In conventional PKI, CAs are assumed to be trusted organisations which verify the identity of the subjects (e.g., domain names) and issue certificates to domains. However, during the last decade, some CAs issued fake but valid certificates for even the well-known domains such as Google, Facebook, Hotmail, GMail, Mozilla, Microsoft $[8,14]$ which could be used to apply MITM kinds of attacks [13].

In order to reduce the ultimate trust to CAs, Google proposed Certificate Transparency (CT) [15] which aims to store the certificates in public logs, so that any certificate which has not been added to the logs would be rejected by TLS clients during certificate validation phase, and a fake certificate could be immediately detected since these logs are publicly visible and monitored by all the related parties. However, CT does not propose any new mechanism for revocation transparency, and relies on the conventional methods such as Certificate Revocation List and Online Certificate Status Protocol [11, 22]. Moreover, it uses multiple log maintainers which reduces usefulness of transparency, since a domain owner has to check each of them for his fake certificates.

Afterwards several other public log based PKI architectures such as Accountable Key Infrastructure (AKI) and Distributed Transparent Key Infrastructure (DTKI) have been proposed to solve these issues [10, 30]. AKI handles common certificate operations including catastrophic events such as domain key loss or compromise by distributing the accountability to new introduced entities. Each entity monitors and reports the operations performed by the other entities. In DTKI, each public log is only responsible for an associated set of domain, and the existence and the revocation status of a certificate can be monitored by either one of these logs or its mirrors.

Recent studies $[5,13,24,27]$ show that blockchain seems to be a promising technology to eliminate the trust to the public logs by decentralizing their management.

\subsection{Our Contributions}

In this paper, we first revisit one of the most recent blockchain-based proposals for PKI (i.e., the Yakubov et al.'s scheme), and address its security and privacy issues within their certificate validation architecture during TLS handshake. More concretely, TLS clients can easily be deceived by fraudulent full nodes or web services during certificate validation, because they cannot verify the validity of the incoming responses. Besides, fake but valid certificates also 
cannot be detected since only the hash values of the certificates are stored in the blockchain. Finally, a malfunctioning CA may not revoke a compromised certificate in a reasonable timeframe which would allow an attacker to exploit this vulnerability before changing the revocation status of the certificate. In order to eliminate these issues, we improve their scheme and propose a new PKI architecture. In summary, our scheme provides the following features.

- We use Practical Byzantine Fault Tolerance Algorithm (PBFT) [4] as the consensus mechanism where the consensus nodes hold a share of the blockchain signing key and a block can only be generated if a threshold number of them approve the block by signing it by their partial key share [21]. Since we use a dynamic threshold signature scheme, once the TLS clients receive the blockchain verification key (i.e., public key) they will not require to change it even if the set of validators is dynamic.

- During TLS handshake, TLS clients can validate the certificates without requiring to be a peer of the blockchain network. Moreover, they do not need to make any further network connection and query other entities during this process. In this respect, certificate and revocation transparency is now fully provided so that the TLS certificates and their revocation status are publicly monitored. Moreover, the privacy of the TLS clients is fully preserved during certificate validation.

- CAs are not the sole authority to revoke certificates anymore, certificate owners can now also revoke their certificates (if they still possess the private key). Therefore, the risk of not revoking a compromised certificate in a reasonable timeframe by a malfunctioning $\mathrm{CA}$ is minimised.

We implement a prototype ${ }^{1}$ of our proposal on Ethereum, and experiment certificate validation. Our experimental results show that TLS clients can validate certificates efficiently (in constant time) depending on only their processing power and memory. Moreover, TLS handshake overhead is insignificant in our scheme.

\subsection{Roadmap}

In Section 2, we briefly describe the most recently proposed blockchain based PKI architectures, and highlight their drawbacks. In Section 3, we revisit Yakubov et al.'s scheme and elaborate its security and privacy issues. In Section 4, we first describe our motivation to use dynamic threshold signatures based permissioned blockchains in our PKI architecture, and then describe PBFT consensus

\footnotetext{
${ }^{1}$ Our prototype is available on https://github.com/efficient-pki-blockchain.
} 
mechanism using dynamic threshold signatures. Finally, we present our new PKI architecture, what we called KORGAN, which eliminates the highlighted issues and provides a more efficient construction than the existing schemes. We discuss our implementation and experimental results in Section 5, and conclude the paper with future works in Section 7.

\section{Related Work: Blockchain Based PKI Architectures}

\subsection{Blockchain-based Certificate and Revocation Transparency [24]}

Wang et al. in [24] proposed to put all issued TLS certificates and their revocation data (i.e., CRL, OCSP) to the blockchain by their corresponding web servers. Each web server has a publishing key pair which is used to sign transactions. A new publishing key has to be approved by a set of web servers using previously approved publishing keys. In this architecture, each transaction has a validity period and the validity period of a certificate addition transaction is shorter than the lifetime of a certificate. Therefore, a certificate is added to the blockchain several times throughout its lifetime. If a certificate is revoked, then the related OCSP response or CRL is also added to the blockchain in a new transaction. During a TLS handshake, a web server sends the Merkle audit proof (standard Merkle tree proof) of its latest certificate addition transaction to the TLS clients. The TLS clients verify the Merkle proof using the block headers which they receive from the P2P network asynchronously. However, this architecture is subject to MITM kinds of attacks in the period of certificate revocation and certificate addition transaction expiration time since certificate addition transaction can be still valid and used for certificate validation even though the certificate is revoked [13].

\subsection{CertChain: Public and Efficient Certificate Audit based on blockchain for TLS Connections [5]}

CertChain [5] proposes a certificate management framework to publicly and efficiently audit TLS certificates on a blockchain. In order to eliminate centralization problems of proof-of-work based consensus mechanisms [18, 25], the authors introduce a new consensus protocol based on Ouroboros [12] which incentivizes CAs and the miners for their honest behaviour. In this mechanism, they introduce a new transaction structure which makes possible to search the history of certificates without sequential traversal of all the blocks. Even though CertChain proposes to find the revocation status of a certificate efficiently through the bloom filters, it is not clear how the implementation of the bloom filters fit to its transaction structure. TLS clients in CertChain ask the 
validity of the certificates to the miners. They have to rely on their responses which can make them subject to MITM kinds of attacks [13]. Moreover, the privacy of the TLS clients is not preserved during the revocation query [28].

\subsection{CertLedger: A new PKI model with Certificate Transparency based on Blockchain [13]}

The authors in [13] propose a new PKI architecture where all the TLS certificates are validated and stored in the blockchain. The entire certificate revocation process and trusted certificate management are also conducted in the blockchain. TLS clients are light nodes of the blockchain network and store block headers to make a successful TLS handshake. However, becoming a peer of the blockchain network brings overhead in terms of storage and network communication for many of the TLS clients.

\subsection{A Blockchain-based PKI Management Framework [27]}

Recently, in [27], Yakubov et al. proposed a new blockchain based PKI architecture for issuing, revoking, and validating X.509 certificates. In this architecture, certificate lifecycle is managed through smart contracts ${ }^{2}$ on the blockchain. Namely, after issuing a new certificate, CA generates a new transaction to add the certificate to its smart contract. Upon validation of the transaction by the consensus nodes and generation of a new block comprising the new transaction, the hash of the certificate is added to an issuance list in the CA smart contract. To revoke a certificate, $\mathrm{CA}$ adds its hash value to a revocation list in the smart contract in a similar fashion. More concretely, a CA smart contract stores an array for all its issued certificates' hash values, a map for the revoked certificates which are referenced by the certificates' hash values, and the CA certificate itself. Clients can validate the certificates through either sending requests to web services or triggering certificate validation smart contract. The CA smart contract is created in such a way that its methods can only be triggered by its owner CA.

\section{Security and Privacy Analysis of the Yakubov et al.'s Scheme}

In this section, we first briefly describe the Yakubov et al.'s Scheme and then point out its security and privacy issues.

\footnotetext{
${ }^{2}$ A smart contract [23] is a self enforcing digital application which contains data and an immutable code to manage it. It can be triggered through transactions in the blockchain.
} 


\subsection{High-Level Description of the Yakubov et al.'s Scheme}

In this scheme, each CA has a dedicated smart contract for issuing and revoking certificates in the blockchain. More concretely, a CA smart contract contains the following features:

- certList $:=\left\{<\right.$ index $_{i}$, certHash $_{i}$, hashAlg $_{i}$, date $\left._{i}>: 1 \leq i \leq \alpha\right\}$ where index $x_{i}$ is the auto generated index for the $i$-th certificate, certHash is the hash value of the $i$-th issued certificate using hash $\lg _{i}$, date $e_{i}$ is the addition date of the certificate to the blockchain, and $\alpha$ is the number of certificates.

- revocationMap $:=\left\{\left\langle\right.\right.$ index $_{i}$, revokeDate $\left.\left._{i}\right\rangle: 1 \leq i \leq \alpha\right\}$ where index $_{i}$ is the index of $i$-th certificate in the certList, revokeDate is $_{i}$ the revocation date of the $i$-th certificate, and $\alpha$ is the number of certificates.

- Its CA certificate $\mathrm{Cert}_{C A}$.

Once a certificate is issued, the CA adds the hash value of the certificate to certList. Similarly, to revoke a certificate, CA adds the index of the certList and the revocation date to the revocationMap. The certificates in this proposal basically comprise several custom X.509 extensions such as CA key identifier and Issuer CA identifier:

- CA key identifier is populated with the CA smart contract address in the CA certificates.

- Issuer CA identifier stores the smart contract address of the issuer of a certificate. This extension is populated for all the certificates apart from the root CA certificates. In fact, it is used for building a trusted path and finding the smart contract address of the issuer of a certificate during the certificate validation.

Certificate validation can be performed in two different methods.

- In the first scheme, the certificate validation algorithm is implemented in a smart contract. This smart contract triggers all the CA smart contracts in the trusted path of a certificate. It validates the existence and the revocation status of all the certificates within this path. This scheme can only be triggered through a full node of the blockchain.

- In the second scheme, certificate validation is delegated to a web service. The web service queries the revocation status of all certificates in the trust chain one by one from a full node.

According to the experimental results, the performance of the second certificate validation scheme has a higher performance than the first one for the trust chains up to 400 sub-CAs. We depict the TLS system architecture with these certificate validation schemes in Figure 1. 


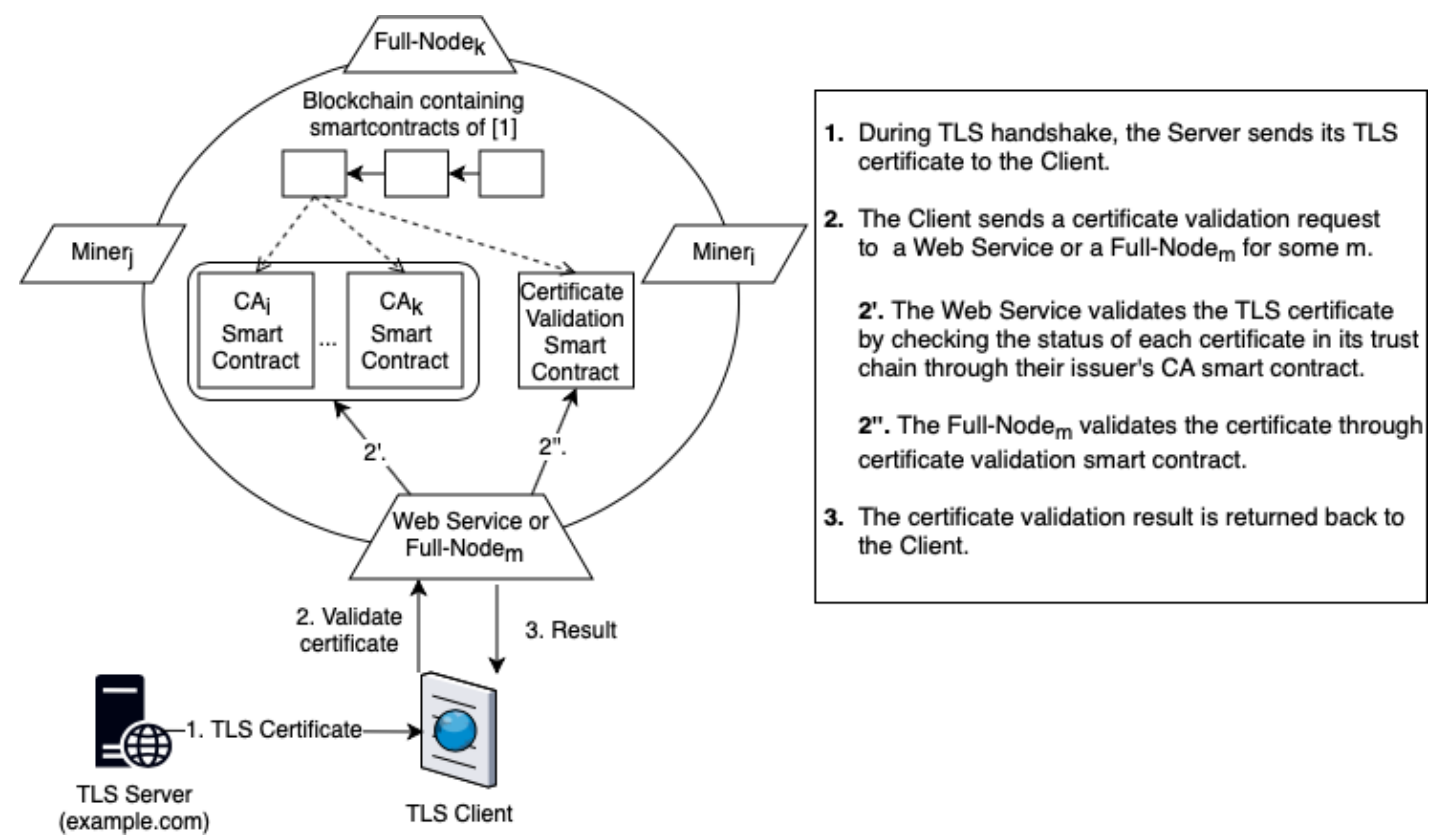

Fig. 1: The TLS System of Yakubov et al.'s Scheme

\subsection{Fake but valid certificates cannot be identified}

The transparency of the certificates is not fully provided in Yakubov et al.'s scheme since only certHash ${ }_{i}$ s are stored in the CA smart contracts. Since it is infeasible to derive the subject of a certificate from certList, it would not be possible for a domain owner to identify and revoke a fraudulent certificate. Consequently, during a TLS handshake, clients could accept fake but valid certificates allowing MITM kinds of attacks [13].

\subsection{Certificates may not be revoked in case of corrupted CAs}

CAs may have to revoke their issued certificates for several reasons such as key compromise or information change (e.g., DNS name). However, if a CA is compromised or does not have a proper revocation process, the certificate may not be revoked in a reasonable time frame or may not be revoked at all. This CA dependent process makes the TLS clients vulnerable to MITM kinds of attacks between the compromise and the revocation time of the certificate [13]. 


\subsection{Certificate validation services can be compromised}

The proposed certificate validation schemes (described in Section 3.1) are subject to MITM kinds of attacks. TLS clients have to rely on either the full nodes or the web service, and trust their certificate validation responses, since these responses do not contain any cryptographic proofs of correctness. Hence, clients can easily be deceived if the full nodes ${ }^{3}$ or web services are corrupted.

Building the trust chain of a certificate is one of the critical components of certificate validation. This process is not fully clarified in the proposal which may be a cause of MITM attacks as well. Namely, creation of CA smart contracts on the blockchain is not subject to authorization, therefore an adversary can deploy a smart contract for a malicious CA which stores fraudulent certificates issued by this fake CA. In this case, TLS clients could be subject to MITM kinds of attacks since they are going to validate these fake certificates through the proposed certificate validation schemes [13].

\subsection{A privacy issue while certificate validation}

As said before, TLS clients cannot validate the certificates themselves (trivially, if they are not a full node of the blockchain). In that case, they have to query a full node or a web service for this purpose. However, this process is not also privacy preserving since these intermediary entities can track the web addresses visited by the TLS clients.

\section{Our Proposal: KORGAN}

\subsection{Our Motivation: Why Dynamic Threshold Signatures based Permissioned Blockchains?}

In order to eliminate the security and privacy issues mentioned in Section 3, TLS clients should be able to use a publicly available blockchain which would include all issued certificates as well as their status without relying any external parties during a certificate validation process. However, this introduces an extra overhead for both permissionless and permissioned blockchains since they have to first verify the validity of the blocks (or only the headers) [26]. More concretely,

\footnotetext{
${ }^{3}$ Full nodes do not execute a transaction which updates the state of the blockchain while running certificate validation smart contract, but only queries blockchain data. Therefore, its malicious behaviour does not have any impact on the blockchain.
} 
- In case of permissionless blockchains, there is going to be a significant network overhead for the TLS clients because they need to be a peer of the blockchain network to determine the valid blocks due to the underlying consensus mechanism [12, 18, 25].

- In case of permissioned blockchains, they have to query a certain number of consensus nodes (i.e., $2 N+1$ in PBFT [4] which requires $3 N+1$ replicas to tolerate $N$ Byzantine failures). This requirement would also become a burden with the increasing number of consensus nodes.

Therefore, becoming a peer of the blockchain network or querying consensus nodes would going to be infeasible for many of the TLS clients due to the limited storage capacity, processing power, or low bandwith. In order to eliminate this overhead, we require authentic blocks which would enable TLS clients to verify their validity efficiently. However, permissionless blockchains are unfortunately not suitable for generating signed blocks because any peer could join the blockchain network and could generate a new block which makes determination of a signature (private) key and distribution of the verification key to the TLS clients infeasible. On the other hand, permissioned blockchains would be more suitable for generating signed blocks since only a limited number of consensus nodes are authorized to generate the new blocks. Still the following requirements must be satisfied for an efficient and scalable solution:

1. Management of the verification key should be a convenient for the TLS clients. Namely, after they receive an authentic verification key they should not change it frequently.

2. There must be only one signature on a block to be optimally scalable.

3. The verification key should not be also changed with addition or removal of the varying number of consensus nodes (i.e., in case of adding new nodes or removing the existing ones). Otherwise, all TLS clients must subsequently update the verification key which would also make the system practically infeasible.

To tackle these requirements, we propose to use dynamic threshold signature schemes among the consensus nodes for signing the new blocks [21]. As in a typical threshold signature scheme, there is going to be only one public key (for verifying a signature) of the overall system, and the private key shares will be owned and managed by the corresponding consensus nodes. If at least a threshold number of consensus nodes agree on a block, then they are going to sign the new block with their private key shares to generate a valid signature.

We highlight that Facebook Libra uses a BFT based consensus algorithm which also utilizes threshold signatures [2, 3, 29], however, their solution does 
not propose dynamic versions of threshold schemes which would incur significant overhead to our scheme. This is because it does not the meet the abovementioned third requirement, and a change in the underlying consensus nodes would result in an update in the clients' public (verification) keys. Thanks to the authors of [21], we have efficient threshold schemes which indeed meet all the requirements and ensure that the remaining consensus nodes are able to add new consensus nodes or remove the corrupted nodes efficiently through only updating their secret shares without changing the overall verification key.

\subsection{Our Approach: PBFT Consensus Mechanisms with Dynamic Threshold Signatures}

The seminal Practical Byzantine Fault Tolerance (PBFT) algorithm aims to reach consensus through Byzantine nodes that tolerates Byzantine failures with low overhead [4]. In particular, PBFT basically uses state-machine replication and replica voting for changing the state in the network. All nodes acting as validators ${ }^{4}$ have equal votes, and validation is executed through multiple rounds to reach the consensus. PBFT utilizes digital signatures to ensure the authenticity of the messages. Nodes have to verify all the signatures received from their peers during each phase of the consensus rounds.

In our blockchain architecture, we utilize a dynamic threshold signature scheme on the PBFT consensus mechanism, and a valid block can only be generated if at least $t$ out of $\ell$ consensus nodes sign the new block [21]. A key generation setup for threshold signature scheme is going to be executed among the predefined consensus nodes as follows:

Threshold Key Generation Setup Among Consensus Nodes We assume that an existing PBFT blockchain with $3 N+1$ consensus nodes has been already setup [4]. Namely, the key generation ceremony will be completed using the underlying PBFT consensus mechanism. More concretely,

1. Each $i$-th consensus node randomly chooses its secret key share $S K_{i}$ and executes the threshold key generation steps (like in [21]), and publishes their intermediate outputs on the blockchain (as a transaction). In particular, the consensus nodes use the underlying blockchain as a public bulletin board to publish and retrieve the necessary data to execute the key generation setup properly.

\footnotetext{
${ }^{4}$ Trivially, as in any permissioned blockchain, we require the validators to be selected from political and geographical disparate entities.
} 
2. Each consensus node queries the blockchain until all the consensus nodes publish their intermediate outputs. A key generation setup would be completed only after all the pre-defined consensus nodes participate to the ceremony ${ }^{5}$. If the steps are validated through the consensus mechanism, $(P K$, $\left.\left(S K_{1}, \cdots, S K_{3 N+1}\right)\right)$ become the verification key and the signing key share of the $i$-th consensus node, respectively.

3. Once all the consensus nodes participate to the key generation ceremony, the selected leader constructs the overall public key and adds as a new transaction.

Once the key generation ceremony is completed successfully, the consensus nodes (i.e., the signers) will only generate signed blocks.

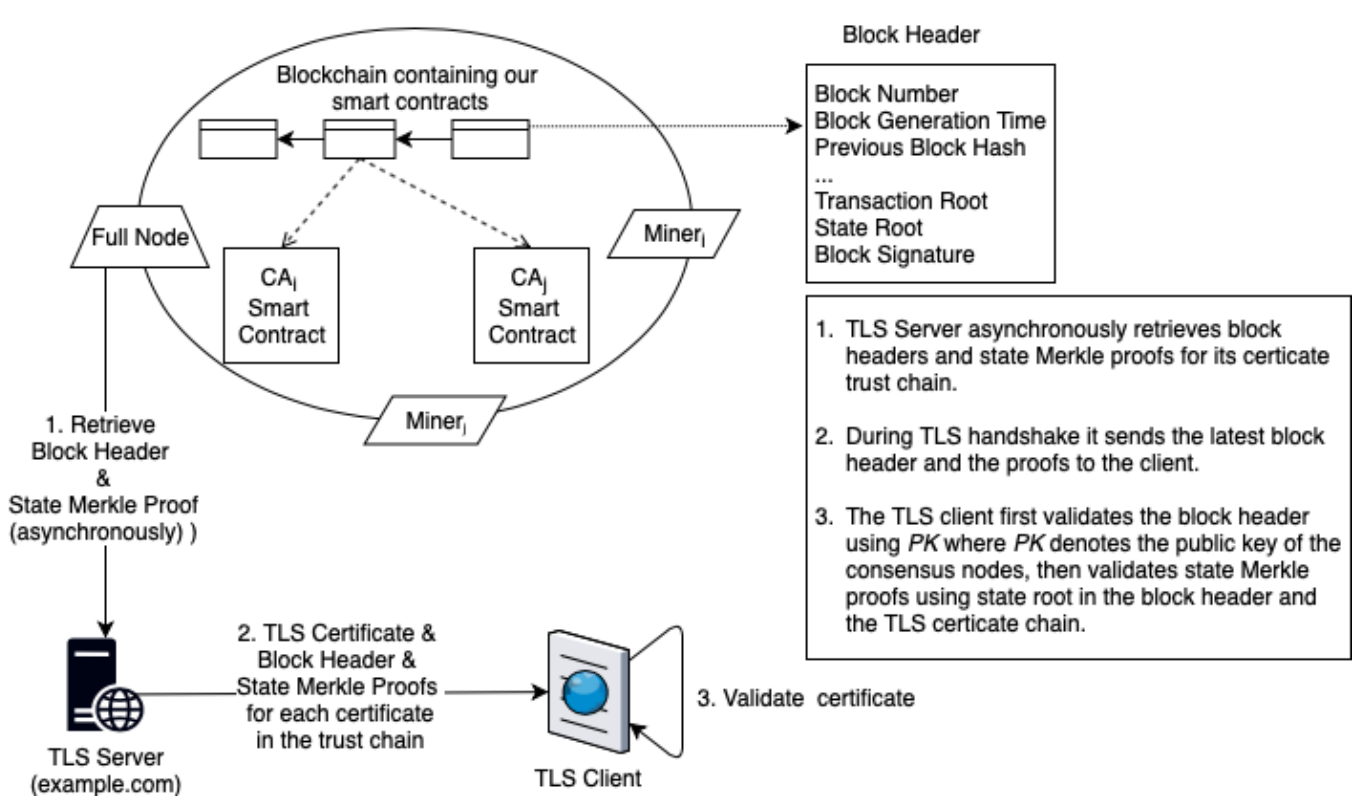

Fig. 2: The TLS System of KORGAN

Block Generation through PBFT with Dynamic Threshold Signatures We now have a dynamic threshold signature scheme on top of PBFT. Note that the underlying consensus mechanism would run in rounds with one node acting as

\footnotetext{
${ }^{5}$ If they do not take part in the ceremony, they will not be able to send signed messages during the consensus phase.
} 
a leader and others as validators. In order to sign a new block, the consensus nodes (i.e., the signers) are going to execute the following threshold signature generation ceremony to generate a valid block.

- Pre-prepare phase: The goal of this phase is to ensure that a majority of honest nodes has agreed on the number of the block being processed for a leader's request. In this flow, the leader initiates the consensus process by sending its partially signed PRE-PREPARE message (using $\operatorname{Sign}_{S K_{i}}$ ) which is a block proposal containing a certain number of transactions.

- Prepare phase: Upon receiving the PRE-PREPARE message, every node in the consensus group checks the correctness and the validity of the block, and multicasts its partially signed PREPARE message (using $S i g n_{S K_{i}}$ ) to all the other nodes.

- Commit phase: Based on the received PREPARE messages, each node combines $t$ signatures, computes a valid signed message, and then multicasts a signed COMMIT message (i.e., "YES/NO") to the consensus group. The new block is committed to the blockchain only if a valid signature is generated.

At the end of the commit phase, all honest nodes in the consensus group would have the same view regarding to the state of blockchain by either accepting or rejecting the block proposal, thereby achieving the confirmed transaction. Consequently, the authenticity of a block (or a block header) can easily be verified by any TLS client using the verification key $P K$. Since the underlying consensus mechanism relies on PBFT, up to $N$ nodes could suffer from Byzantine failure. Therefore, at least $t=2 N+1$ out of $3 N+1$ nodes should be trustworthy to preserve the same security level of the underlying PBFT.

Adding and Removing Consensus Nodes Upon addition and removal of the consensus nodes, private key shares of all the consensus nodes has to be updated without changing the overall $(P K, S K)$ as described in [21]. Furthermore, the threshold $t$ has to be updated in such a way that it has to preserve the same security level of the underlying PBFT.

Assume that we have $3 N+1$ consensus nodes and $t=2 N+1$. If we want to add (or remove) $3 m$ nodes, then we have in total $3(N+m)+1$ (or $3(N-m)+1$ in case of removal) nodes. Then, $t$ must become $2(N+m)+1$ (or $2(N-m)+1$ in case of removal) to reach the same security level of PBFT. 


\subsection{The CA Smart Contract in KORGAN}

As in the Yakubov et al's scheme, in KORGAN, we assume that CAs validate the domains (i.e., ACME protocol [1] for DV SSL Certificates ${ }^{6}$ ) using the processes described in their certificate policy and certificate practice statement documents, and issue the certificate afterwards. In the following, we will describe the blockchain structure and the CA smart contracts in KORGAN.

We use the permissioned blockchain with the PBFT consensus mechanism (described in Section 4.2) which allows TLS clients to easily verify the final state of a certificate. To enable this, consensus nodes maintain a State Merkle Tree $(\mathrm{SMT})^{7}$ which is used to store and verify the state of all accounts, smart contract codes and the data within the smart contracts. SMT is updated with each block according to the block transactions and its Merkle root is put into the generated block header. A sample block header of KORGAN's architecture is depicted in Figure 2. Since all the certificates and their revocation status are stored in the CA smart contracts, the state of each certificate can also be tracked in every block. Moreover, their state can be verified using the Merkle proof generated from the SMT and the Merkle root. Since Merkle root is stored in the signed block header and its authenticity can be verified by $P K$, TLS clients can easily check the validity of a certificate without relying any parties during TLS handshake.

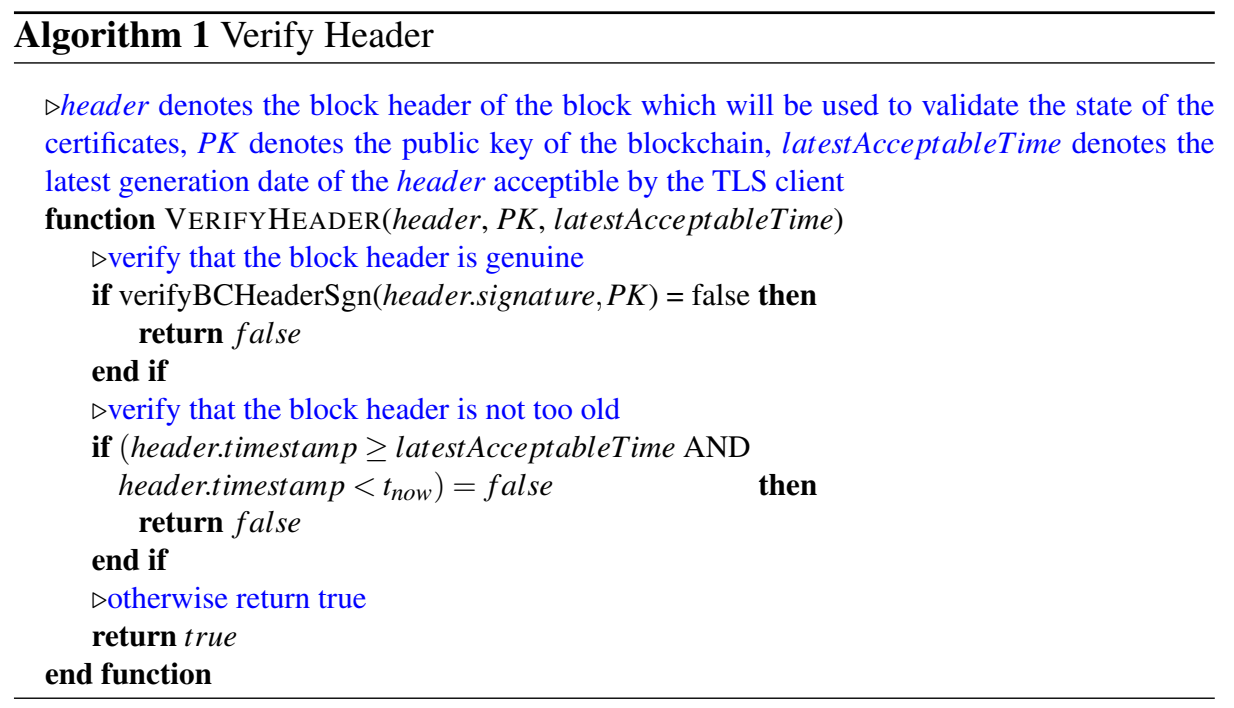

\footnotetext{
${ }^{6}$ Domain Validation SSL certificates are issued after proving the right to use the domain.

${ }^{7}$ Modified Merkle Patricia Tree [9] can be used for this purpose where search, insert and update operations can be performed in logarithmic time.
} 
Instead of an array with certificate hash values and a map for the revoked certificates, KORGAN modifies the CA smart contract in the Yakubov et al.'s scheme by storing the following two maps:

- The first map is certURIMap $:=\left\{\left(\right.\right.$ uri $\left._{i},<\operatorname{certHash}_{i, j}>\right): 1 \leq i \leq \alpha, 1 \leq$ $j \leq \beta\}$ where $u r i_{i}$ is the $i$-th certificate subject (or subject alternative name), certHash $_{i, j}$ is the hash of the $j$-th certificate of the $i$-th uri, and $\alpha, \beta \in \mathbb{N}^{+}$. This map provides the transparency of the certificates, and the domain owners can use it to monitor ${ }^{8}$ the blockchain if a fraudulent certificate is issued for their web servers.

- The second map is used to track the revocation status of the certificates and represented as certRevocationMap $:=\left\{<\operatorname{certHash}_{k}, r s_{k}>: 1 \leq k \leq\right.$ $\gamma\}$ where $\operatorname{certHash}_{k}$ is the $k$-th certificate hash, $r s_{k}$ is the revocation status ("revoked", "valid") of the $k$-th certificate, and $\gamma \in \mathbb{N}^{+}$.

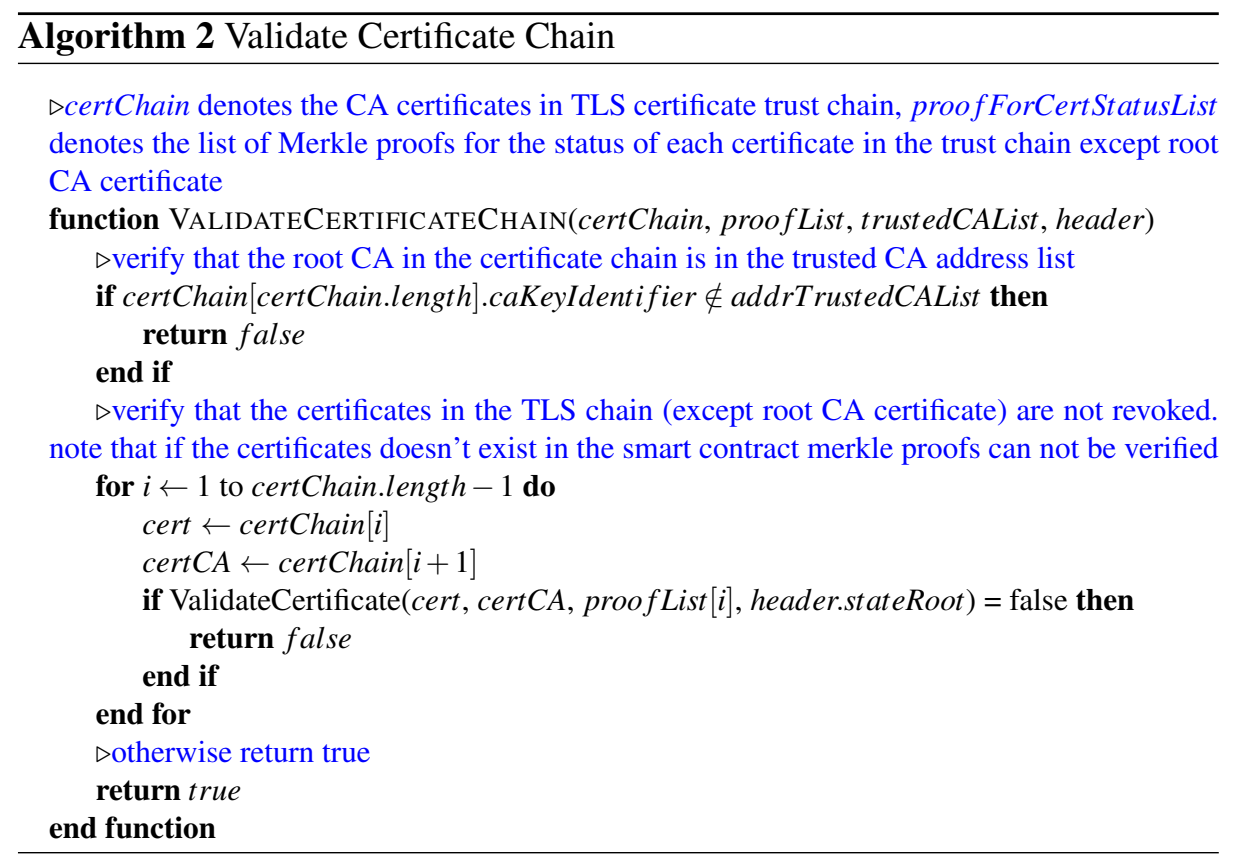

In our CA smart contract, a certificate can only be added to the blockchain by its issuing CA. However, the status of the certificate can be changed as "revoked" by both its issuing CA and owner. A certificate owner can only trigger

\footnotetext{
${ }^{8}$ Event listeners can be used for this purpose which triggers certain events (e.g., SMS, e-mail etc.) in case a certain condition is satisfied in the smart contract.
} 
the revocation method of the smart contract if he can prove his ownership to the certificate. Therefore, this method requires a signature generated by the private key of the certificate.

Note that block confirmation time must be short enough to discourage adversaries to perform a MITM kind of attack during block time. In this respect, due to the underlying PBFT mechanism and dynamic threshold signature scheme, our consensus scheme provides high throughput and low transaction latency similar to LibraBFT [16] which meets our requirements.

\subsection{Certificate Validation of KORGAN in SSL/TLS}

TLS clients are not required to be full or light nodes of the blockchain, thus do not have to retrieve any blocks (or headers) from the blockchain network. For certificate validation, they only need to store $P K$ to verify the authenticity of the block headers and the trusted root CAs' smart contract addresses to construct the trust chain. On the other hand, TLS servers have to periodically retrieve the block headers and the Merkle proofs associated with their TLS certificate chain from a full node of the blockchain. The retrieval process is independent of the TLS handshake and can be conducted asynchronously.

KORGAN does not change the TLS handshake protocol but introduces new TLS extensions to be used during the ServerCertificate step of the protocol (see Figure 2). In these extensions, a TLS server sends the latest block header, certificate chain, and a list of Merkle proofs for the revocation status of each certificate in the chain to the TLS client. A TLS client performs the following steps to validate the TLS certificate.

1. Verifies the signature of the block header by $P K$, and reads the authentic block generation time and the SMT root from the block header.

2. Checks whether the block generation time is fresh enough according to its security settings. However the acceptable freshness period should not be shorter than the block time and it should not reject the latest block header.

3. Checks whether the TLS certificate is issued from a trusted root CA by searching the smart contract address (CA key identifier) of the root CA in its trusted list.

4. Validates each certificate in the trust chain by validating the Merkle proofs using the certificate's hash value, its issuing CA's smart contract address (Issuer CA identifier) and the SMT root.

5. Checks the revocation status of each certificate in the trust chain (except root CA certificate) and verifies that none of them are revoked.

The first and second step of our certificate validation algorithm is described in more detail in Algorithm 1, third step in Algorithm 2, and finally fourth and 


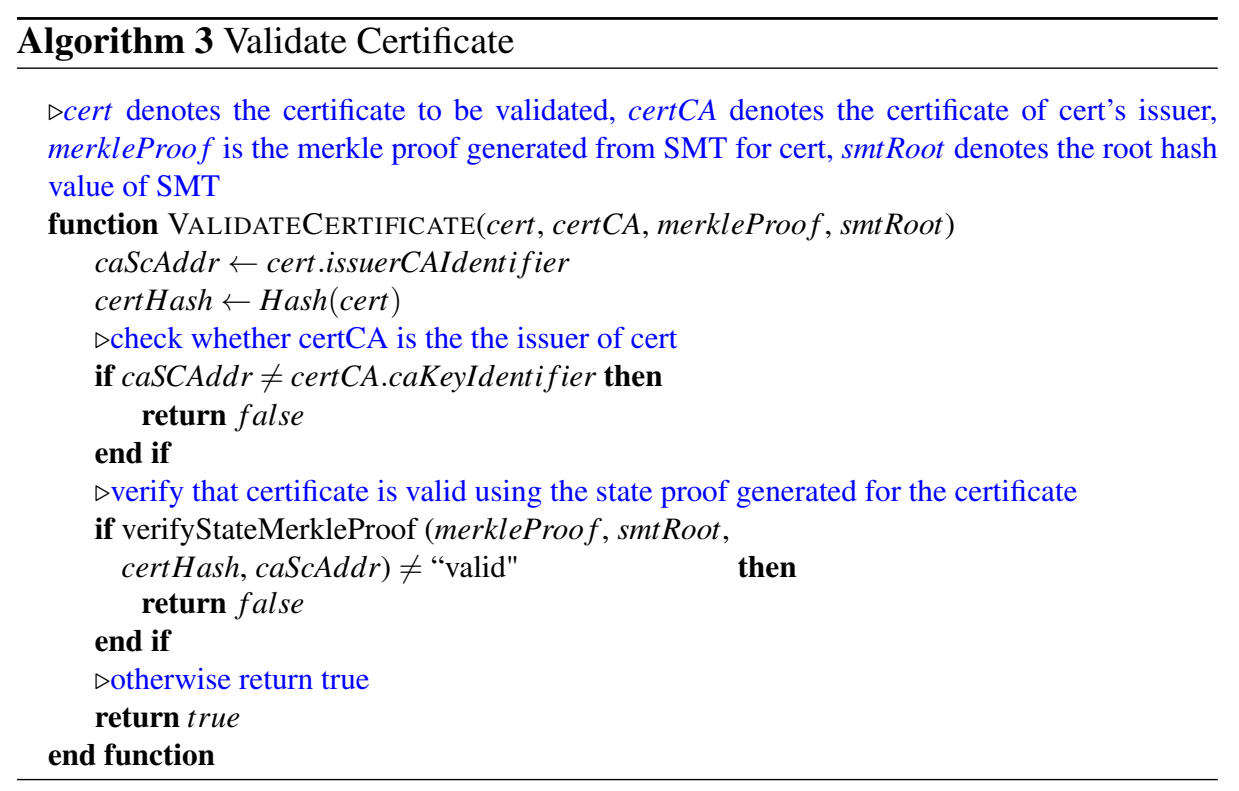

Table 1: TLS Handshake Experimental Results

\begin{tabular}{|c|c|c|c|c|}
\hline $\begin{array}{c}\text { Number of TLS Certificates } \\
\text { in the CA Smart Contract }\end{array}$ & $\begin{array}{c}\text { Header Data Size } \\
\text { (bytes) }\end{array}$ & $\begin{array}{c}\text { Account Proof Data Size } \\
\text { (bytes) }\end{array}$ & $\begin{array}{c}\text { Storage Proof Data Size } \\
\text { (bytes) }\end{array}$ & $\begin{array}{c}\text { TLS Handshake Overhead Total } \\
\text { (bytes) }\end{array}$ \\
\hline 1 & 535 & 758 & 590 & 1883 \\
\hline 100 & 535 & 758 & 1089 & 2382 \\
\hline 1.000 & 535 & 758 & 1557 & 2850 \\
\hline 10.000 & 535 & 758 & 2027 & 3320 \\
\hline
\end{tabular}


fifth steps in Algorithm 2 and 3. We would like to highlight that our algorithm does not require any further network connections, thus the privacy of the TLS clients is also fully preserved. The execution time of the algorithm is not effected from the network latency and only depends on the processing capability of the TLS clients.

\section{Implementation and Experimental Results}

We have implemented KORGAN by updating the CA smart contract of Yakubov et al.'s scheme ${ }^{9}$ [27] and experimented certificate validation with our scheme. For the experiments, we deployed two smart contracts on private Ethereum network so that we had two CAs in the trust chain. For generation and verification of the state Merkle proofs, we used Eth-proof node-js API [31]. We executed our experiments on a Macbook Pro with Intel Core i7 (3.1 Ghz) CPU, 16 GB of memory and macOS Majove OS.

We demonstrate the experimental results for TLS handshake overhead in Table 1 and elaborate them as follows. First, Header denotes the size of the block header in Ethereum, therefore its size is constant and independent of certificates in the smart contract. Second, AccountProof is the proof generated to validate the overall state of the smart contract (i.e., the account) comprising its balance, code, and the stored data. Note that its size grows logarithmically with the number of smart contracts in the blockchain due to its Patricia Tree structure [9]. In our first experiment, there was only one CA smart contract, therefore, the size of the AccountProof size is the same independently of number of TLS certificates. Third, StorageProof is also generated from Storage Merkle-Patricia Tree which is different for all smart contracts in Ethereum. The root value of this tree is also used while computing the state of the account. The size of the StorageProof is $\log _{2} n \times c_{1}+c_{2}$ where $n$ is the number certificates in an account, $c_{1}$ is a constant calculated by adding hash length forming the Merkle proof with the path length between the nodes, and $c_{2}$ is the size of input where its hash is calculated to generate a leaf node in the Merkle tree. Hence, the overall TLS Handshake overhead in our scheme is calculated as

$$
\mid \text { Header } \mid+(\mid \text { AccountProof }|+| \text { StorageProof } \mid) \times n
$$

where $n$ is the number of CAs in the TLS certificate chain.

We note that certificate validation network overhead is not given in Yakubov et al.'s scheme. On the other hand in the conventional PKI, the size of a CRL changes with respect to the number of certificates issued by the CA. Even though

\footnotetext{
${ }^{9}$ https://github.com/snt-sedan/pki-blockchain
} 
Table 2: Certificate Validation Durations Based on \# of TLS Certificates

\begin{tabular}{|c|c|}
\hline $\begin{array}{c}\text { Number of TLS Certificates } \\
\text { in the CA Smart Contract }\end{array}$ & $\begin{array}{c}\text { Certificate Validation } \\
\text { Duration (ms) }\end{array}$ \\
\hline 1 & 55,56 \\
\hline 100 & 56,37 \\
\hline 1.000 & 59,24 \\
\hline 10.000 & 60,45 \\
\hline
\end{tabular}

there are CRLs ranging up to $28 \mathrm{MB}^{10}$, the CRL size for the median certificate is calculated as $51 \mathrm{~KB}$ in [17]. In case of OCSP usage for revocation checking, the average size for an OCSP response is about $\sim 4 \mathrm{~KB}$. Moreover, the total network overhead increases with respect to the length of the trust chain.

Our experimental results in Table $2 \& 3$ demonstrate that the number of certificates in a CA smart contract and the length of the trust chain does not significantly effect the certificate validation duration. On the other hand, in conventional PKI, if the revocation check of a certificate is performed through OCSP, then the latency only due to the network traffic is around $200 \mathrm{~ms}$ [20]. The total duration increases with respect to the number of certificates in the trust chain. Since the size of CRLs are much bigger than OCSP responses, their average downloading latency is also greater then OCSP [19].

Table 3: Trust Chain Length (number of CA smart contracts*)

\begin{tabular}{|c|c|}
\hline $\begin{array}{c}\text { Trustchain Length } \\
\text { (number of CA smart contracts*) }\end{array}$ & $\begin{array}{c}\text { Certificate Validation } \\
\text { Duration (ms) }\end{array}$ \\
\hline 1 & 59,24 \\
\hline 2 & 59,85 \\
\hline 3 & 60,13 \\
\hline 5 & 60,67 \\
\hline
\end{tabular}

* There are 1000 certificates in each smart contract.

${ }^{10}$ Apple hosts $28 \mathrm{MB}$ of CRL at http://crl apple.com/wwdrca.crl 


\section{Discussion}

In our proposal, we require a dynamic threshold signature scheme to keep the verification key unchanged in the TLS clients, and preserve the same security level of PBFT independently from adding and removing a consensus node. It is clear that the change in the number of consensus nodes does not affect the faulty assumption of PBFT which does not exceed 33\%. Since the nodes in PBFT are stateless, once a node is removed (which cannot be a part of the consensus mechanism anymore) it will not be able to have an impact on the consensus.

However, in our proposal, it is probable that removed nodes can become active and colluding adversaries (considering dynamic adversaries instead of static adversaries). Since each node possesses a partial private key share $S K_{i}, t$ compromised nodes (with possibly colluding removed ones) still will be able to use their $S K_{i}$ s to generate a fake block by creating a valid signature without running a consensus protocol. Therefore, the acceptable level of the threshold $t$ could be changed dynamically. To make the system $\alpha$-tolerant where $\alpha<t$, we accept to remove $\alpha$ nodes without changing the $(P K, S K)$ key pair. However, if more then $\alpha$ nodes are removed, $(P K, S K)$ should be updated and the new $P K$ should be distributed to the TLS clients. We would like to highlight that the removed nodes not necessarily become colluding adversaries but for the sake of security we assume that $P K$ should be changed once $\alpha$ nodes have been removed. The number $\alpha$ can be adjusted according to security considerations, however we do not expect this removal frequently as the nodes are chosen from reputable organizations like universities, large world-wide companies (like Google), IETF, IEEE, and NIST.

\section{Conclusion and Future Work}

There have been recent serious security incidents due to misbehaving CAs which have issued fraudulent certificates. To make CAs more transparent, various public log based and blockchain based PKI models are proposed. In this paper, we point out security and privacy issues of one of the most recent proposals (belonging to Yakubov et al.) and eliminate them by proposing a new PKI architecture, what we called KORGAN. KORGAN is based on permissioned blockchain and utilizing PBFT where the blocks are signed through dynamic threshold signature scheme among consensus nodes. Due to the signed blocks, TLS clients can now easily verify the final states of certificates without requiring to be a peer of the blockchain network. Our experimental results on Ethereum demonstrate that KORGAN does not bring any significant computational and network overhead during certificate validation. Even more, the duration of our certificate validation is less than the previous schemes. 
Further research work mainly includes modifying KORGAN in such a way that generating CA smart contracts could be restricted to only trustworthy CAs. For this purpose, an international board can be established to audit the CAs and sign the smart contract generation transaction using a threshold signature scheme as well. 


\section{Bibliography}

[1] Barnes R, Hoffman-Andrews J, McCarney D (2019) Automatic Certificate Management Environment (ACME). RFC 8555 (Standard)

[2] Boneh D, Lynn B, Shacham H (2001) Short Signatures from the Weil Pairing. In: Advances in Cryptology - ASIACRYPT 2001, Springer, Berlin, Gold Coast, Australia, pp 514-532

[3] Cachin C, Kursawe K, Shoup V (2005) Random Oracles in Constantinople: Practical Asynchronous Byzantine Agreement Using Cryptography. Journal of Cryptology 18(3):219-246

[4] Castro M, Liskov B (2002) Practical Byzantine Fault Tolerance and Proactive Recovery. ACM Transactions on Computer Systems 20(4):398-461

[5] Chen J, Yao S, Yuan Q, He K, Ji S, Du R (2018) CertChain: Public and efficient certificate audit based on blockchain for TLS connections. In: IEEE INFOCOM 2018-IEEE Conference on Computer Communications, IEEE, Honolulu, HI, USA, pp 2060-2068

[6] Conti M, Dragoni N, Lesyk V (2016) A Survey of Man In The Middle Attacks. IEEE Communications Surveys Tutorials 18(3):2027-2051

[7] Dierks, T and Rescorla, E (2018) The Transport Layer Security (TLS) Protocol Version 1.3. RFC 8446 (Proposed Standard)

[8] DigiNotar public report (2012) Black tulip report of the investigation into the DigiNotar certificate authority breach. Fox-IT

[9] Ethereum (2019) Patricia Tree. https://github.com/ethereum/wiki/ wiki/Patricia-Tree

[10] Hyun-Jin Kim T, Huang L, Perrig A, Jackson C, Gligor V (2013) Accountable key infrastructure (AKI): A proposal for a public-key validation infrastructure. In: Proceedings of the 22nd international conference on World Wide Web, Association for Computing Machinery, New York, USA, Rio de Janeiro, Brazil, pp 679-690

[11] ITU-T X509 (2012) Information Technology-Open Systems Interconnection-The Directory: Public-Key and Attribute Certificate Frameworks. International Telecommunications Union, Geneva, Switzerland

[12] Kiayias A, Russell A, David B, Oliynykov R (2017) Ouroboros: A Provably Secure Proof-of-Stake Blockchain Protocol. In: Advances in Cryptology - CRYPTO 2017, Springer International Publishing, Santa Barbara, CA, USA, pp 357-388 
[13] Kubilay MY, Kiraz MS, Mantar HA (2019) CertLedger: A New PKI Model with Certificate Transparency Based on Blockchain. Computer \& Security 85:333-352

[14] Langley A (2015) Maintaining digital certificate security. https://security.googleblog.com/2015/03/ maintaining-digital-certificate-security.html

[15] Laurie, B, Langley, A and Kasper, E (2013) Certificate Transparency. RFC 6962 (Experimental)

[16] Libra (2019) LibraBFT Consensus Performance. https://developers . libra.org/docs/crates/consensus

[17] Liu Y, Tome W, Zhang L, Choffnes D, Levin D, Maggs B, Mislove A, Schulman A, Wilson C (2015) An end-to-end measurement of certificate revocation in the web's PKI. In: Proceedings of the 2015 Internet Measurement Conference, Association for Computing Machinery, New York, USA, Tokyo Japan, pp 183-196

[18] Nakamoto S (2008) Bitcoin: A Peer-to-Peer Electronic Cash System. https://bitcoin.org/bitcoin.pdf

[19] Netcraft (????) NetCraft. CRL Sites in September 2019. https:// uptime .netcraft.com/up/reports/performance/CRL

[20] Netcraft (2019) NetCraft. OCSP Server Performance in September 2019. https://uptime.netcraft.com/up/reports/performance/OCSP

[21] Noack A, Spitz S (2009) Dynamic Threshold Cryptosystem without Group Manager. Network Protocols \& Algorithms 1(1):108-121

[22] Santesson S (2019) X.509 Internet Public Key Infrastructure Online Certificate Status Protocol - OCSP. RFC 6960 (Standard)

[23] Szabo N (1997) Formalizing and Securing Relationships on Public Networks. https://firstmonday.org/ojs/index.php/fm/article/ view/548

[24] Wang Z, Lin J, Cai Q, Wang Q, Jing J, Zha D (2019) BlockchainBased Certificate Transparency and Revocation Transparency. In: Financial Cryptography and Data Security, Springer, Nieuwpoort, Curaçao, pp $144-162$

[25] Wood G (2014) Ethereum: A Secure Decentralised Generalised Transaction Ledger. http://gavwood.com/paper.pdf

[26] Wüst K, Gervais A (2018) Do you need a blockchain? In: 2018 Crypto Valley Conference on Blockchain Technology (CVCBT), IEEE, Zug, Switzerland, pp 45-54

[27] Yakubov A, Shbair WM, Wallbom A, Sanda D, State R (2018) A blockchain-based PKI management framework. In: NOMS 2018 - 2018 IEEE/IFIP Network Operations and Management Symposium, IEEE, Taipei, Taiwan, pp 1-6 
[28] Yao S, Chen J, He K, Du R, Zhu T, Chen X (2019) PBCert: PrivacyPreserving Blockchain-Based Certificate Status Validation Toward Mass Storage Management. IEEE Access 7:6117-6128

[29] Yin M, Malkhi D, Reiter MK, Gueta G, Abraham I (2019) HotStuff: BFT Consensus with Linearity and Responsiveness. In: Proceedings of the 2019 ACM Symposium on Principles of Distributed Computing, Association for Computing Machinery, New York, USA, Toronto, Canada, pp 347-356

[30] Yu J, Cheval V, Ryan M (2016) DTKI: A New Formalized PKI with Verifiable Trusted Parties. The Computer Journal 59(11):1695-1713

[31] Zac M (2019) Eth Proof 2.0.0. https://github.com/zmitton/ eth-proof 